Cahiers de recherches médiévales

\title{
Historiciser les paroles de personnages de bande dessinée
}

L'exemple de la série de Hermann, Les Tours de Bois-Maury

Corinne Denoyelle

\section{(2) OpenEdition \\ Journals}

\section{Édition électronique}

URL : https://journals.openedition.org/crm/5862

DOI : $10.4000 / \mathrm{crm} .5862$

ISSN : 1955-2424

\section{Éditeur}

Honoré Champion

\section{Édition imprimée}

Date de publication : 20 juin 2008

Pagination : 255-262

ISSN : $1272-9752$

Référence électronique

Corinne Denoyelle, "Historiciser les paroles de personnages de bande dessinée », Cahiers de

recherches médiévales [En ligne], 15 | 2008, mis en ligne le 20 juin 2011, consulté le 15 décembre 2022. URL : http://journals.openedition.org/crm/5862 ; DOI : https://doi.org/10.4000/crm.5862 


\section{RM}

\section{Historiciser les paroles de personnages de bande dessinée : l'exemple de la série de Hermann, Les Tours de Bois-Maury}

Le Moyen Âge offre à tous les raconteurs d'histoire un décor et un cadre riche de potentialités dramatiques: une nature encore sauvage, pesant fortement sur les vies humaines, des bâtiments massifs, propres à des mises en scènes décoratives, une violence latente dans les rapports humains, ne demandant qu'à exploser en guerres, viols et rapines, tortures et exécutions. C'est ainsi que Hermann présente sa série les Tours de Bois-Maury sur le site officiel qui lui est consacré : « une époque rude, une violence omniprésente, la nature hostile. Les personnages ont les pieds dans la boue, une espérance de vie très faible, des préjugés, une peur de l'étranger'. » Que la réalité historique ait été plus nuancée importe peu : le Moyen Âge en bande dessinée, c'est essentiellement des jolies filles peu farouches, des cavalcades à cheval, des étripages en règle. Cependant à travers ce cadre général assez sommaire que se donnent les auteurs de bande dessinée, la critique universitaire peut trouver à se déployer et à enquêter. À la suite de Bernard Ribémont ${ }^{2}$, on distinguera diverses catégories qui réinventent pour leurs besoins narratifs un Moyen Âge plus ou moins éloigné de ce que nous connaissons de la réalité historique. On dessinera grossièrement deux tendances dans la bande dessinée à destination des adultes: celle des auteurs qui réinventent un Moyen Âge fantasmé dans le cadre de l'Héroïc Fantasy, que B. Ribémont appelle "médio-fantasmatique», souvent inspiré de Tolkien, et dans lequel la magie vient brouiller les repères historiques. Le succès de ce genre vient de sa liberté extrême qui lui permet toutes les fantaisies. Une autre tendance, à laquelle nous nous intéresserons ici rassemble les auteurs qui cherchent au contraire à coller le plus possible à la vérité historique telle que nous pouvons nous la représenter, catégorie que nous qualifierons «d'historique » selon les termes de B. Ribémont. Le travail de recherche documentaire, graphique et social, très poussé de ces auteurs apparaît dans le scénario, dans les décors, dans le costume et se nuance selon les cas d'une dimension romanesque ou onirique... mais il se heurte presque toujours à la limite des paroles des personnages. Comment faire parler ceux-ci d'une manière qui fasse aussi couleur locale ? Comment éviter l'anachronisme du langage quand on a donné un soin extrême à tout le cadre jusqu'aux boucles des heaumes ?

Umberto Eco, quand il s'est lancé dans la fabuleuse aventure du Nom de la Rose a reconnu qu'il s'agissait là d'un écueil essentiel de tout roman historique :

\footnotetext{
1 http://www.hermannhuppen.com/archives_hh.php3 : Les Tours de Bois Maury : Présentation de la saga.

${ }^{2}$ http://bernard.ribemont.neuf.fr/chantierBD.htm

Il distingue cinq catégories de bandes dessinées médiévalisantes : les séries simples pour les enfants; les séries strictement éducatives; les séries «historiques» (historico-épiques ; historico-romanesques; historico-oniriques); les séries médio-fantasmatiques et les séries médio-dérisionnelles.
}

Cahiers de Recherches Médiévales, 15, 2008 
Les dialogues me posaient un autre problème. Jusqu'à quel point pouvaient-ils être médiévaux ? En d'autres termes, je me rendais compte, à l'écriture, que le livre prenait une structure de mélodrame bouffe, avec de longs récitatifs et d'amples arias. Les arias (la description du portail par exemple) se référaient à la grande rhétorique de l'Âge Moyen, et là les modèles ne manquaient pas. Mais les dialogues ? À un moment donné, j'ai craint que les dialogues ne soient de l'Agatha Christie quand les arias étaient du Suger ou du Saint Bernard ${ }^{3}$.

On peut le déplorer, mais nul n'est tenu de lire couramment l'ancien français, ni les lecteurs de bande dessinée, ni leurs auteurs. En général, ils semblent ne pas trop se poser la question et limitent leur historicisation des paroles de personnages à quelques jurons ou termes techniques. Parfois, quelques-uns utilisent l'espace de liberté spécifique qu'offre l'insertion de chansons dans le cadre du récit. Là peut se déployer une langue plus datée, plus poétique et détachée des exigences narratives. C'est ce que fait Servais dans le superbe Isabelle, ou, dans un registre comique, Delporte écrivant pour Peyo les meilleurs scénarios de Johan et Pirlouit et empruntant pour ce «troubadour ${ }^{4} »$ des extraits de fabliaux médiévaux. Certains auteurs plus scrupuleux se sont efforcés d'inventer une langue originale pour leurs héros, qui réponde à la double exigence de la compréhension et de l'exotisme, qui sonne «médiévale» sans offrir trop d'obstacles à la lecture. Cet idiome original, qui recherche un effet d'oralité, se doit d'avoir des niveaux de langue appropriés à la classe du locuteur (qu'il soit vilain, clerc ou grand seigneur). La démarche la plus complète et la plus aboutie de cette recherche est celle de François Bourgeon dans sa formidable série des Compagnons du Crépuscule ${ }^{5}$. Bourgeon a utilisé le dictionnaire d'ancien français d'A. J. Greimas chez Larousse, qu'il a complété par la lecture de nombreux textes médiévaux, en particulier des farces, et il a choisi environ trois cents mots et expressions qui colorent la langue de ses personnages. Michel Thiebaut, dans une vaste étude ${ }^{6}$ de ses «romans graphiques », a fait une analyse complète et détaillée du résultat obtenu. L'auteur s'est essentiellement appuyé sur le lexique qu'il a enrichi de termes de métier (le bassinet à mézail pour désigner le casque du chevalier) de préfixes expressifs (esforcier; malmort), de raccourcis (peurpisser) ou de transpositions grammaticales (péagier, culeter). L'essentiel de son travail porte sur le vocabulaire, plutôt dépréciatif, désignant les corps masculins et surtout féminins, les jurons et les injures. Il a moins touché à la syntaxe, se limitant essentiellement aux effets obtenus par l'omission de l'article ou par celle du

\footnotetext{
${ }^{3}$ Umberto Eco, Apostille au Nom de la Rose, traduit de l'italien par Myriem Bouzaher, in Le Nom de la Rose, Paris, Grasset, 1985, pp. 509-544, p. 523.

${ }^{4}$ Le terme est toujours utilisé improprement. Pour une étude approfondie de Pirlouit et de ses autres collègues, voir F. Plet, «Le Jongleur. Portrait de l'artiste en clown» in Le Moyen Âge par la bande, Études de Lettres, 2001, 1. Articles recueillis par Alain Corbellari et Alexander Schwarz, Université de Lausanne, 2001, pp. 37-64.

${ }^{5}$ François Bourgeon, tome 1. Le Sortilège du Bois des Brumes, Casterman, 1986; Les Yeux d'étain de la ville glauque, Casterman, 1988 ; Le dernier chant des Malaterre, Castermann, 1990.

${ }^{6}$ Michel Thiebaut, Dans le Sillage des sirènes, autour des Compagnons du Crépuscule de François Bourgeon, Casterman, 1992, pp. 21-28
} 
sujet. Il modifie quelque peu l'emploi des prépositions : privilégiant à aux dépens de de qu'il n'hésite pas à omettre. Il déplace le pronom complément devant l'auxiliaire : "Torneirie qui me voudrait voir morte» (Le Dernier chant des Malaterre, p. 87) et l'adjectif épithète devant le substantif. Comme le note M. Thiebaut : «Cette langue est surtout évocatrice», riche de connotations. «Le plaisir de la lecture se double alors d'une sorte de jeu de devinettes» qui tend par moment à l'hermétisme. Aucun autre auteur, à ma connaissance, n'est allé aussi loin.

Toute autre, mais tout aussi intéressante, est la démarche de Hermann, l'auteur des Tours de Bois-Maury, auquel je voudrais m'intéresser ici plus longuement. Cette série, que l'on peut qualifier d'historico-épique dans la mesure où elle est calquée sur le modèle de la chanson de geste, raconte les aventures du chevalier Aymar de Bois-Maury, dans la quête qu'il mène pour retrouver le domaine de son enfance dont il a été dépossédé, thème ancien et classique dans la littérature épique s'il en est. L'histoire est censée se passer à la fin du $\mathrm{XI}^{\mathrm{e}}$ siècle, à l'époque de la première croisade. La quête est suffisamment vague pour servir de prétextes à des voyages et à des rencontres qui sont le véritable support dramatique du récit. En fait, on voit surtout Aymar rêver à son domaine mais ce n'est que dans le dixième album de la série, qu'il entreprend de le reconquérir concrètement. Le chevalier mourra cependant avant de revoir les tours de Bois-Maury. Par ailleurs, il a souvent moins d'importance narrative que quelques personnages de paysans, d'ouvriers ou de voleurs dont on suit de près les aventures et qui réapparaissent d'albums en albums. Enfin, une dimension onirique est parfois apportée par des épisodes merveilleux sur lesquels cependant l'auteur ne s'attarde pas.

Hermann prétend ne pas avoir cherché à véritablement historiciser la langue de ses personnages : «L'emploi sporadique de l'ancien français donne une pointe d'exotisme, mais cela relève plus de l'emballage narratif que de la recherche linguistique pointue. À cette période, nombreux étaient les dialectes locaux, cela aurait demandé une recherche énorme et fastidieuse pour un résultat improductif : le lecteur d'aujourd'hui s'y serait certainement perdu'. » Son travail sur la langue n'est volontairement pas aussi complexe que celui de Bourgeon, il ne cherche pas à donner à ses personnages une langue fondamentalement différente de la nôtre mais juste à la marquer discrètement et à lui donner un léger «parfum » d'ancienneté selon ses propres termes. Inspiré par la lecture des minutes des procès de Jeanne d'Arc, Hermann s'est aussi aidé du dictionnaire de Greimas, et a eu le plaisir de retrouver pour ses personnages quelques mots du dialecte wallon qu'il a pratiqué enfant. Le résultat est plus subtil et plus précis qu'il ne le prétend et est révélateur d'une certaine conception du Moyen Âge.

Les marques qu'il utilise pour signifier l'ancienneté de son récit sont essentiellement de nature lexicale et syntaxique. De rares effets phoniques s'y ajoutent. Elles sont diversement distribuées selon les personnages et selon leur classe sociale. $\mathrm{Au}$ niveau social, une nette hiérarchie est assurée entre le héros, noble chevalier errant au langage châtié et respectueux, maniant sans peine l'imparfait du subjonctif, et les autres personnages dont la langue est moins marquée.

Les déformations phoniques sont réservées aux paysans, technique déjà utilisée à l'âge classique, que l'on songe aux personnages de Molière: les vilains sur

${ }^{7}$ http://www.hermannhuppen.com/ Article intitulé : Bois-Maury - Le genre humain 
lesquels s'ouvre la série dans le premier tome Babette, se caractérisent par la chute des $e$ dans quelques mots monosyllabiques: «M'est avis qu'la Babette, elle a de nouveau l'cœur dans les taillis. » (p. 3); «Babette, j'sais point c'qu'elle est devenue !» (p. 19); «J'vas te faire goûter au bâton!» (p. 4). Dans le tome 2, Éloïse de Montgri, un paysan s'étonne : «Et puis y a aussi ce chevalier de Bois-Quéque'chose qu'est parti de son côté.» (p. 34). En faisant disparaître les $e$, Hermann donne aux paysans un parler connoté comme populaire que vient encore renforcer la transformation de bien en ben: «Tu peux courir, va. Faudra ben que tu reviennes à ce soir !» (Babette, p. 3) et des modifications syntaxiques: ellipse du sujet impersonnel, généralisation du déterminant devant le nom propre et emploi généralisé de la préposition à pour les compléments circonstanciels. Ces variations phoniques sont limitées aux personnages les plus bas sur l'échelle sociale, aucun accent étranger n'est noté même quand le chevalier part en croisade et traverse l'Anatolie ou chemine avec un compagnon allemand. Elles sont particulièrement dépréciatives. La jeune amoureuse Babette, victime de la force masculine, ne parle en rien comme son père «Par Dieu, messire, passez votre chemin ou je vais être forcée de vous frapper. » (p.6) dit-elle au jeune seigneur qui tente de la violer; «Il n'est nul besoin d'envoyer vos soldats, mon seigneur, je suis là !» (p. 9) Ce qu'Hermann écrit n'a rien de spécifiquement médiéval jusqu'ici, il dote ses personnages d'un parler connoté comme populaire depuis très longtemps.

Les modifications syntaxiques sont plus discrètes mais aussi plus systématiques et caractérisent tous les personnages, surtout les plus nobles. Hermann s'efforce de retrouver des négations plus archaïques. S'il ne revient pas à une négation simple entièrement portée par le $n e$, il recherche des particules de renforcement qui fassent ancien : le ne...point est quasiment systématique; Aymar de Bois-Maury va plus loin en remarquant que «il se fait tard, on n'y voit plus goutte, et le vent fraîchit» (Babette, p. 13), dans ce cas, il triple la négation : le sens négatif, porté par les adverbes ne et plus, est renforcé par le substantif goutte. Olivier, son écuyer, plus simple que son maitre mais sympathique, emploie des tournures plus modernes et donc moins visibles: «Il n'y a pas de jeune fille dans la chaumière.» (id. p. 19) Les paysans se limitent au deuxième mot: «Ma fille est point rentrée depuis l'jour d'avant. » (id. p. 19)

Les interrogations sont presque tout le temps construites par une inversion du sujet: «Sont-ce gens d'armes là-dehors ou pillards comme-nous ?» (Éloïse p. 41) ; «Verrons-nous le seigneur Eudes nous faire escorte?»(Babette, p. 29) «Que te semble-t-il, Olivier?»; «Est-il de vos gens? » La jeune Babette, plus simple, formule ses interrogations en est-ce que : «Et puis, qu'est-ce que cela change?» (Babette, p. 14), elle ne tombe pas dans la vulgarité d'un mendiant cupide et violent qui s'exclame, ironique: «mais c'est t'y ${ }^{8}$ pas l'ami Germain que j'aperçois là ? » (id., p.44)

Le déterminant est fréquemment omis, suite à des formules de présentation «Voilà présence qui m'apaise» (Germain, p. 24); «Voilà cheminement périlleux qui voit moult ne jamais en revenir.» (id, p. 26); pour des pluriels indéfinis ren-

\footnotetext{
${ }^{8}$ Sur le $t i$ comme marque de l'interrogation, voir F. Godet, Le français standard, p. 109. Elle note que cet élément, aujourd'hui disparu du langage standard reste régional et limité à quelques reproductions artificielles du langage populaire.
} 
voyant à une quantité : «Je perds tout entendement, voilà château frappé de malheur... et malgré cela, cidre, vins, bonne chair et fruits...» (Alda, p. 15) ; «C'est que mêmes cris m'avaient inquiétée» (id. p. 29); pour des noms abstraits : «En quoi avait-il raison de délivrer le chevalier et son écuyer ${ }^{9}$ ?» (id. p. 29), «cette femme a enduré autant que courage peut! Ce serait vilenie de lui faire subir plus !» (Germain, p. 35) ; des locutions : «Je lui proposai mariage» (id, p. 31) ; dans certains cas, on a plus de mal à comprendre ce qui a autorisé Hermann à ne pas mettre de déterminant. Peut-être le sens irréel de la phrase laisse-t-il planer une certaine indétermination sur le substantif : «Je ferai bâtir grande et belle demeure sur la montagne. » (Germain, p. 23) ; «il est plus pesant que cochon!» (Alda, p. 27) dit pour évoquer le poids d'un cadavre.

L'adjectif est fréquemment antéposé : «Je conçois qu'une innocente ardeur aveugle un si jeune enfant.» (Élö̈se, p. 32); «C'est qu'on te voyait dans quelque taillis à mener joyeuse fête en agréable compagnie. » (Germain, p. 12) L'emploi des prépositions est aussi un peu modifié par rapport au français moderne: dans est remplacé par en: "Le sang ne sera pas versé en la demeure de Dieu » (Babette, p. 12), par dedans: «Vous irez dedans l'eau»(Alda, p. 26). La préposition à connaît une extension plus grande de son emploi : «J'avais commencé à trouver plaisance à vivre ici...» (id., p. 43)

La syntaxe du verbe est peu touchée. Le sujet est parfois omis quand la phrase le permet : «Je n'osais en espérer autant, messire Aymar, et vous suis reconnaissant de votre silence. » (Germain, p. 37); «Vous n'en saurez rien et vous enjoins de faire vite. » (Alda, p. 26) ; mais cela reste rare. Hermann déplace parfois le pronom complément devant l'auxiliaire : «Est-ce ton maître qui te commande de saisir l'acier dès qu'un chevalier le vient voir ?» (Alda, p. 11) mais cela n'est jamais systématique puisque quelques lignes plus loin, il fait dire au comte de Portel : « Nul ne vint plus me voir. » (Alda, p. 13) Il use fréquemment d'une sorte de datif d'intérêt placé avant le verbe : «la prière nous est une nourriture » (Babette, p.6); «Et si rien ne nous vient avant le jour?» (Germain, p. 11); «Les jours te sont-ils doux, ici ?» (Babette, p. 43); «S'il y avait péril, ces hommes nous viendraient autrement!» (Alda, p. 11) ; «Il faut que je vous entretienne de choses qui me sont importantes.» (Élö̈se, p. 32). L'exemple suivant cumule ces phénomènes dans une proposition infinitive : « Bois-Maury... J'aime à $m$ 'y penser vous rendant visite. » Par extension, le pronom complément peut recevoir une construction directe avec des verbes transitifs indirects : «Il me semble avoir ouï quelques ris... Me moquerait-on?» (Alda, p. 10)

Les principaux marqueurs syntaxiques qui connotent l'ancienneté de la langue se trouvent dans l'emploi important des constructions impersonnelles ou passives, plus fréquentes dans l'ancienne langue jusqu'à l'époque classique que dans notre français standard contemporain : «Il m'est un grand déplaisir de vous écouter en parler en mal ! » (Babette, p. 8) ; «Il n'est nul besoin d'envoyer vos soldats. » (id.

\footnotetext{
${ }^{9}$ Cet exemple est assez subtil car il se rapproche de l'expression avoir raison, opposée à avoir tort, mais ce n'est pas de cela qu'il s'agit ici, on devrait avoir en français moderne : «avoir une raison d'agir ainsi ou ainsi». Cette formulation n'est pas médiévale mais en se superposant avec la première dans l'esprit du lecteur, elle prend un aspect inhabituel qui peut passer pour ancien.
} 
p. 9); «Il me revient seulement d'avoir entendu des voix.» (id. p. 9); « Il me tarde vous voir en tournoi. » (id. p.39) «Il m'est bien dur d'oublier les chagrins de messires Eudes et de sa dame.» (id. p. 39)) «Il serait injuste de vous en faire reproche [...] mais il me sera désormais cruel d'avoir à croiser votre regard.» (id. p. 29) Le passif reçoit un développement remarquable : «Et point de gibet n'y sera vu pour pendre des paysans. » (Babette, p. 13); «Des frères nous ont été ôtés.» (id. p. 38). Ces constructions entraînent une extension de l'emploi du verbe être au lieu d'avoir: «Est-il un maçon du nom de Germain?» (Babette, p. 11). En utilisant ces expressions, Hermann fait preuve d'une intuition et d'une connaissance de l'ancienne langue assez fine.

L'impression d'ancienneté qui se dégage des paroles de personnages tient donc essentiellement à la construction verbale, à l'importance des formulations impersonnelles ou passives, aux interrogations par inversion du sujet, complétées par l'emploi des pronoms à valeur de datif d'intérêt, l'omission de quelques déterminants. Tout ceci fournit à la langue des personnages un squelette relativement stable sur lequel vient se greffer un lexique archaïsant.

C'est en effet celui-ci qui porte essentiellement le poids des connotations anciennes : Hermann emploie très peu le vocabulaire technique spécifiquement médiéval. Alors que d'autres séries caractérisent la langue de leur personnages uniquement par ce biais au point d'être obligé de le préciser par des notes de bas de page ${ }^{10}$, l'auteur n'insiste pas sur les termes d'habillement ou d'armement, néglige les vassal, suzerain, félon, adoubement, homme-lige... qui font traditionnellement partie des représentations que l'on a des hommes et de la langue du Moyen Âge. Il préfère mettre en avant des termes encore connus aujourd'hui dont la forme seule a un peu changé.

Quelques exclamations ou jurons colorent les propos des personnages: $T u$ dieu, Par Dieu, Las, Diable, par l'enfer... là encore sans attirer sur eux une visibilité extrême. Hermann remplace quelques unes des particules adverbiales oui/non par non point, que non, que oui, de fait, certes quand il s'agit d'une personne bien née... Soit est mis pour signifier l'accord. Par ailleurs, il utilise certains adjectifs ou certains substantifs uniquement sous la forme ancienne du mot : vivant est systématiquement remplacé par vif (Alda, p. 16), fou par fol-follet pour le nom (Alda, p. 28)-, anneau par anel, (Alda, p. 22). D'aucuns est utilisé pour quelques-uns : «Ce serait un assassin que d'aucuns appellent le maçon» (Alda, p. 6); «D'aucuns en parlent mais jamais il ne me fut donné d'en rencontrer» (Germain p. 20); «D'aucuns en feraient de même» (id. p. 30) Dans la plupart des cas, l'auteur reprend un terme ancien, voire disparu, mais dont une forme dérivée a subsisté, ce qui permet de préserver la compréhension du lecteur moderne : conter est ainsi mis à chaque fois pour raconter. On trouvera aussi, des termes plus rares : pour les verbes, notons disparoir pour disparaître: «Si tu restes là à regarder le soleil disparoir... » (Alda, p. 25), mirer pour regarder: "Cela fait un moment que je vous mire» (Le Seldjouki, p. 14); défuir : «Oui, cher Aymar! le silence. Jusqu'à mes voisins qui me défuyent.» (id. p. 13), encourir pour courir « Hé, toi! Ne t'encours pas. De quoi astu peur? » (Babette, p. 40) une maisonelle renvoie à une chaumière (Germain, p. 20 ou Alda p. 1) Pour les adjectifs, on remarquera le rare voisinal pour désigner par

${ }^{10}$ Voir la série Les Aigles décapités par exemple. 
exemple «la geôle voisinale» dans laquelle croupissent depuis de nombreuses années le fils et l'épouse du comte de Portel, à côté de celle où sont tombés le chevalier de Bois-Maury et son écuyer (id. p. 24). En dehors des suffixes en -aille qui restent fréquents, « la vile rascaille» (Alda, p. 4), la mangeaille (Alda, p. 24); les épousailles (id, p. 45), Hermann privilégie les suffixes en -ance ou en -ement qui donnent un style très châtié à ses personnages : «Je n'en ai pas souvenance » (Babette, p. 43); «J'avais commencé à trouver plaisance à vivre ici... » (Alda, p. 35); «je vous remercie toutefois de nous rappeler la veillance» (id. p. 23) «Cette désordenance la tient depuis qu'un homme sans honneur lui fit un enfant.» (Germain, p. 36) ; «J'ai commandement de vous mener à lui sur l'heure ; (Alda, p. 18); «ce serait allègement de les savoir pendus » (id. p. 16).

Dans d'autres cas, il reprend un mot ancien, disparu : moult, grand classique du style «troubadour» est fréquemment utilisé comme pronom : «elle est femme et sait mieux que moult comment écarter le danger» (Germain, p. 19)»; «voilà cheminement périlleux qui voit moult ne jamais en revenir.» (id. p. 26), plus rarement comme adjectif «il y a là moult brins de laine pris dans les buissons.» (Élö̈se, p. 35) ; «Nous reviendrons moultes fois le regarder ensemble.» (Alda, p. 45). Céler est mis pour cacher «Mais, il n'est point seul! Que cela cèle-t-il ?» (Germain, p. 18) ; ire pour colère «Rentre ta ire !»(Germain, p. 9); «Je me suis laissé porter par la ire !» (Alda, p. 32), ouïr pour entendre «je crois avoir ouï hennir les chevaux; (Eloüse, p. 32) «Oyez tous ces coups de tonnerre», (id p. 7); dextre pour main droite : "Et si le malheur lui a ôté l'usage de la main gauche, la dextre en vaut plus que deux. » (Germain, p. 7); «À ta dextre, dans l'ombre, des yeux s'attachent à nous. » (Alda, p. 16) alors que main gauche n'est jamais remplacée. Le verbe chaloir remplace le verbe s'intéresser à : «Tailler la pierre te chaut» (Germain, p. 7); «Votre hardiesse me chaut, messire Aymar! Souffrez que ce soit moi qui vous fende la panse. » (Alda, p. 43); le verbe seoir remplace convenir à : « cet atour de gente dame ne te sied plus » (Alda, p. 35) ; «cette explication me sied davantage...» (Babette, p. 7) ; occire remplace tuer «Nos gardes sont occis» (Élö̈se, p. 6); «Il nous occira sitôt après » (Germain, p. 38) ; quérir est mis pour chercher : «Va quérir les compagnons et conduits les à Bois Renan. » (Alda, p. 16) choir pour tomber qu'il s'agisse d'un oiseau : «Tu l'as frappé! Regarde comme il vole mal, il va choir!» (Germain, p. 5) ou d'un objet «Ne laisse rien choir de ton sac...» (id., p. 18); gésir est mis pour coucher : «Je vais te montrer où gésir avec d'autres qui ont cherché refuge chez nous; » (id. p.12), advenir (parfois avenir) remplace arriver: «Si ces deux hommes ne sont pas rattrapés, tu sais ce qui nous avendra!» (Alda, p. 29) ; «Que vous est-il advenu?» (id. p. 39) ; entendre remplace comprendre : «J'y perds mon entendement - Je n'y entends pas davantage. » (Élö̈se, p. 9) mitan est mis pour milieu: «Elle nous arriva un jour au mitan de bateleurs à l'étrange portement. » (Alda, p. 24); «Et si nous ne les trouvons pas avant le mitan du jour?» (id. p. 29). On notera aussi l'utilisation irrégulière de quiet pour tranquille, de férir pour frapper, de bouter pour mettre (bouter le feu), de honnir pour maudire... Plus surprenant, Hermann utilise le mot sotie, pour désigner une erreur (Alda, p. 28). Ce sens, connu au Moyen Âge, n'est plus attesté en français moderne, il s'appuie sans doute là sur la proximité phonique et étymologique avec la sottise. C'est le seul cas qui peut passer pour hermétique à un lecteur normalement cultivé. Dans plusieurs exemples, l'image vient suppléer ce que le mot peut avoir de difficile 
et le contexte immédiat apporte aussi une signification: «Je sais seulement que les arondes volent trop bas pour qu'il n'y ait pas de pluie demain...» constate Olivier en regardant les hirondelles voler au soleil couchant. (Alda, p. 13).

Certains mots, encore existant aujourd'hui, sont utilisés dans un sens disparu : souffrir est mis pour permettre: «Souffrez que je fasse abstinence, monseigneur;»(Babette, p. 7) D'autres termes sont pris dans leur sens classique: ainsi méchant pour mauvais, employé pour une chose : "Tout cela n'est qu'un méchant prétexte pour quitter cette demeure ; (Babette, p. 22) ; «Vous n'aurez plus assez de temps pour ruminer ces méchants souvenirs » (id. p. 39); fort est utilisé comme adverbe d'intensité : "J'incline fort à penser que la femme de notre marchand se portera mieux » (Germain, p. 44); «Il est vrai que les derniers tournois m'ont fort éprouvé » (Babette, p. 12).

Nous conclurons en reprenant la comparaison que nous avions initiée au départ entre le travail linguistique de François Bourgeon et celui de Hermann: dans les Compagnons du Crépuscule, le Moyen Âge est un monde haut en couleurs, certes cruel aux faibles et aux petits, certes fanatique et violent, mais aussi d'une sensualité extrême, avide de jouissances jusque dans les mots. Il délivre tout le charme d'un monde chatoyant, pittoresque, où la langue, moins bridée et moins policée que la nôtre possède une véritable matérialité phonique et sémantique. Chez Bourgeon, le souci du détail vestimentaire, architectural, linguistique est emblématique de la charge affective que porte pour lui le Moyen Âge. De même que la répétition litanique inlassable des Séries redonne vie aux anciennes traditions, de même il veut insuffler la vie dans ces vieilles pierres et ces vieux mots.

Chez Hermann, le Moyen Âge nous est moins étranger de nature, hommes et femmes nous y ressemblent plus. La magie n'affleure plus derrière chaque rocher. Il est le cadre de la confrontation des destins, symbolisés par les noms que chaque personnage important donne à un album. Hommes et femmes se croisent, se séparent, se retrouvent, s'affrontent et se confrontent. Ils sont le premier centre d'intérêt de la série, plus que le dépaysement ou l'exotisme d'une époque. À ce titre les premières planches de cette série sont emblématiques, dans leur atemporalité, de cette volonté de rendre compte de toute une époque sans y rester limité : elles mettent en scène la jeune Babette et sa famille occupées aux travaux des champs, et hormis la forme de la fourche et de la carriole, ou l'harnachement du bœuf qui conduit l'attelage, rien ne connote particulièrement le Moyen Âge, ni n'enferme les personnages dans une époque. Si Hermann s'appuie sur une documentation solide, il ne se laisse pas aller à la séduction de cette civilisation et la langue finalement plus classique que strictement médiévale qu'il donne à ses héros participe à cette volonté de les élever vers une dimension universelle. Il se concentre sur les âmes auxquelles il offre vie : qu'ils soient enlisés dans la boue du pays de Caux ou écrasés par le soleil brûlant de l'Aquitaine, ses personnages n'ont jamais rien de bas ni de vil. Pauvresses, mendiants, larrons, chevaliers sans terre... leur dignité éclate dans leurs paroles. La langue travaillée et châtiée qu'ils parlent leur donne à tous noblesse d'âme et grandeur épique.

Corinne Denoyelle

CELAM/CETM -Université de Rennes II 